\begin{tabular}{|c|l|}
\hline Title & Direct observation of nuclear field fluctuations in single quantum dots \\
\hline Author(s) & Kaji, R.; A dachi, S.; Sasakura, H.; Muto, S. \\
\hline Citation & $\begin{array}{l}\text { Physical Review B, 85(15), 155315 } \\
\text { https://doi.org/_0.1103/PhysRevB.85.155315 }\end{array}$ \\
\hline Issue Date & 2012-04_15 \\
\hline Doc URL & http://hdl.handle.net/2115/49142 \\
\hline Rights & @2012A A merican Physical Society \\
\hline Type & article \\
\hline File Information & PRB85-15_155315.pdf \\
\hline
\end{tabular}

Instructions for use 


\title{
Direct observation of nuclear field fluctuations in single quantum dots
}

\author{
R. Kaji, ${ }^{1, *}$ S. Adachi, ${ }^{1}$ H. Sasakura, ${ }^{2}$ and S. Muto ${ }^{1}$ \\ ${ }^{1}$ Department of Applied Physics, Hokkaido University, N13 W8, Kitaku, Sapporo 060-8628, Japan \\ ${ }^{2}$ Research Institute for Electronic Science, Hokkaido University, N21 W10, Kitaku, Sapporo 001-0021, Japan
}

(Received 27 August 2011; revised manuscript received 13 February 2012; published 13 April 2012)

\begin{abstract}
The spin interaction between an electron and nuclei was investigated optically in a single self-assembled InAlAs quantum dot (QD). In spin dynamics at the initial stage, the fluctuation of nuclear field and the resulting electron spin relaxation time play a crucial role. We examined a positively charged exciton in a QD to evaluate the key physical quantities directly via the temporal evolution measurements of the Overhauser shift and the degree of circular polarization. In addition, the validity of our used spin dynamics model was discussed in the context of the experimentally obtained key parameters.
\end{abstract}

DOI: 10.1103/PhysRevB.85.155315

PACS number(s): 73.21.La, 78.67.Hc, 71.35.Pq, 71.70.Jp

\section{INTRODUCTION}

The hyperfine interaction in semiconductor quantum dots (QDs) is enhanced owing to the strong 3D confinement of the electron wave function; consequently, this has attracted considerable attention from the fundamental and practical points of view. The sophisticated control of nuclear spin polarization (NSP) is required for fascinating applications such as a long-lived memory at an atomic level ${ }^{1}$ and qubit conversion between an electron spin and a photon. ${ }^{2}$ In semiconductor QDs, the enhanced hyperfine interaction provides the possibility of polarizing nuclear spins (n-spins) in one direction with the optically selective excitation of the electron spin (e-spin). In fact, a large NSP of up to 30-60\% was observed recently in interface GaAs QDs, ${ }^{3}$ self-assembled InAlAs QDs, ${ }^{4-6} \operatorname{In}(\mathrm{Ga})$ As QDs, ${ }^{7-9}$ and InP QDs. ${ }^{10}$ In these QDs, the confined electron is subject to a large nuclear field (Overhauser field: $B_{\mathrm{N}}$ ) up to several teslas. From this point of view, it is necessary to examine the spin dynamics of a coupled electron-nuclei (e-n) system that is well isolated in a QD.

Another fundamental interest is the knowledge of the e-spin dephasing induced by the random fluctuation of the Overhauser field. It has been known that spin relaxation of a QD-confined electron, at low temperature, is mainly due to the e-n hyperfine interaction. ${ }^{11-14}$ For a QD-confined electron, its correlation time during which the Overhauser field is considered to be constant (frozen) is quite longer than the e-spin precession period, and therefore the e-spin coherently precesses around the effective magnetic field which is composed of an externally applied magnetic field and the Overhauser field. However, the Overhauser field composed of a large number of $n$-spins $\left(\sim 10^{4}\right)$ inherently has the random fluctuations of its magnitude and direction, and a key parameter $\Delta B_{\mathrm{N}}$ is used as a measure of the degree of $B_{\mathrm{N}}$ dispersion. ${ }^{11}$ Considering an ensemble of individual QDs, the dispersion of $B_{\mathrm{N}}$ induces the difference of the e-spin precession frequency and the resulting e-spin dephasing of the QD ensemble within a characteristic time $T_{\Delta}=\hbar /\left(g^{\mathrm{e}} \mu_{\mathrm{B}} \Delta B_{\mathrm{N}}\right)$, where $g^{\mathrm{e}}$ and $\mu_{\mathrm{B}}$ denote an electron $g$ factor and Bohr magneton, respectively. This apparent macroscopic dephasing occurs even for an e-spin confined in a single QD by sampling and averaging over the ensemble of measurements. Thus $\Delta B_{\mathrm{N}}$ imposes an inevitable contribution to the e-spin relaxation (i.e., the decay of the longitudinal component of the e-spin polarization). $T_{\Delta}$ is one of the significant parameters which determine the coherence of the confined e-spin, and therefore the experimental evaluations of $\Delta B_{\mathrm{N}}$ and resulting $T_{\Delta}$ are very important.

A secondary interest is the possibility of the measurement of NSP in a QD structure through the degree of circular polarization (DCP) of positively charged exciton emissions. The DCP of the time-integrated photoluminescence (PL) has been used as a powerful tool to detect NSP or the Overhauser shift (OHS), which is the energy shift in the electronic level induced by $B_{\mathrm{N}}$, in bulk and quantum well structures for a long time. ${ }^{15}$ However, the method to probe NSP in single QDs has been limited only to the change in the energy splitting of the PL lines; this is the simplest way to evaluate OHS, but its accuracy has been limited by the spectral resolution of the experimental setup. In the coupled e-n system, by using the DCP of positively charged exciton emission, which directly reflects the e-spin polarization, it may be possible to follow not only the e-spin but n-spin dynamics, and the study of the coupled e-n system may act as a tool for sensitive measurements of QD-NSP.

In this study, we investigated the e-n spin dynamics in QD structures by using the DCP of the positively charged exciton $\left(\mathrm{X}^{+}\right)$. The DCP of $\mathrm{X}^{+}$PL changed in synchronization with the OHS or the energy splitting of the e-spin levels, and this phenomenon provides the possibility of the sensitive probing of the QD-NSP. By taking advantage of this feature, the key quantities $\left(\Delta B_{\mathrm{N}}\right.$ and $\left.T_{\Delta}\right)$ were evaluated directly from the experimental data. In addition, we extended the dynamics model of NSP by including the dynamics of the $\mathrm{X}^{+}$states, and we confirmed the validity of the e-spin relaxation model by comparing the time-resolved OHS and DCP measurements with the calculated results.

\section{SAMPLE AND SETUP}

The self-assembled QD sample used in the experiments was grown on an undoped (100) GaAs substrate in the Stranski-Krastanow growth mode by molecular beam epitaxy. An $\operatorname{In}_{0.75} \mathrm{Al}_{0.25} \mathrm{As}$ QD layer was embedded in $\mathrm{Al}_{0.3} \mathrm{Ga}_{0.7} \mathrm{As}$ layers, and a GaAs cap layer terminated the heterostructure. More details of the sample preparation can be seen in previous works. ${ }^{16,17}$ By atomic force microscopy measurements of a reference uncapped sample with the same growth condition, the average QD diameter of $20 \mathrm{~nm}$, height of $4 \mathrm{~nm}$, as well 
as areal density of $5 \times 10^{10}$ dots $/ \mathrm{cm}^{2}$ were found. Assuming a lens-shaped QD with the typical diameter and height, the number of nuclei in a single QD was estimated roughly to be $\sim 3 \times 10^{4}$. To isolate a single QD, small mesa structures were fabricated by electron-beam lithography and wet chemical etching. From the mesas with the typical top lateral size of $150 \mathrm{~nm}$, the well-separated sharp emissions appeared by conventional far-field spectroscopy.

Micro-PL measurements were performed at $6 \mathrm{~K}$ under longitudinal magnetic fields $\left(B_{\mathrm{z}}\right)$ of up to $5 \mathrm{~T}^{18} \mathrm{~A} \mathrm{cw}-$ Ti:sapphire laser beam traveling along the $\mathrm{QD}$ growth direction was focused on the sample surface by using a microscope objective $(\times 20, \mathrm{NA}=0.4)$. The excitation wavelength was tuned to $\sim 728 \mathrm{~nm}$ which provides the transition energy at the wetting layer of the QDs. The QD emission spectra were detected using a triple-grating spectrometer and a liquid- $\mathrm{N}_{2}$ cooled Si-CCD detector. Though the energy resolution of our setup was $\sim 12 \mu \mathrm{eV}$, it can be improved to $5 \mu \mathrm{eV}$ by spectral fitting. The polarization of the excitation light was controlled using a quarter wave plate for the steady-state measurements (Sec. III A) and an electro-optic modulator (EOM) for the time-resolved measurements (Sec. IIIC), and the Zeeman splitting energy and the DCP of the target single-QD spectra were evaluated.

\section{RESULTS AND DISCUSSION}

\section{A. Electron and nuclear spin polarization in a coupled system}

First, we investigate the availability of $\mathrm{X}^{+} \mathrm{DCP}$ as a powerful measure of the electron and nuclear spin polarizations in a QD. In this section, we performed the steady-state PL measurements. Figure 1(a) shows the PL spectra obtained from the target single QD under a zero magnetic field. Regardless of the nominally undoped sample we used, the PL spectra from the various charge states that originated from the same single QD were observed. By using a variety of methods, e.g., from the exciton fine-structure measurements, ${ }^{19}$ magneto-PL measurements under transverse magnetic fields,${ }^{20}$ the autocorrelation of PL, ${ }^{21}$ and the binding energy consideration, ${ }^{22}$ the charge states of three peaks with high intensities were assigned to a neutral biexciton $\left(\mathrm{XX}^{0}\right)$, a neutral exciton $\left(\mathrm{X}^{0}\right)$, and a positively charged exciton $\left(\mathrm{X}^{+}\right)$from the lower energy side, respectively. In the lowest $\mathrm{X}^{+}$state composed of the spin-paired two holes and an electron, the spin-flipped electron gets the optically allowed transition and can recombine with a hole irrespective of its spin direction. Furthermore, the hole spin can easily flip during the energy relaxation process from the wetting layer to the QD ground state. This feature is advantageous to achieve the rapid NSP formation cycle compared to the neutral exciton that has the dark states with a long lifetime. In addition, since $\mathrm{X}^{+}$has the strongest PL intensity in our target $\mathrm{QD}$, it is expected to be the dominant contributor in NSP formation.

Figure 1(b) depicts the $\mathrm{X}^{+}$PL spectra at $B_{\mathrm{z}}=5 \mathrm{~T}$ for the linearly (denoted by gray squares) and the circularly ( $\sigma_{+}$and $\sigma_{-}$: denoted by open and solid circles) polarized excitations. In the $\mathrm{X}^{+}$states, the exchange interactions between the electron and hole spins play no role, and the energy splitting of the PL lines $\left(\Delta E_{\mathrm{Z}}\right)$ is determined solely by the
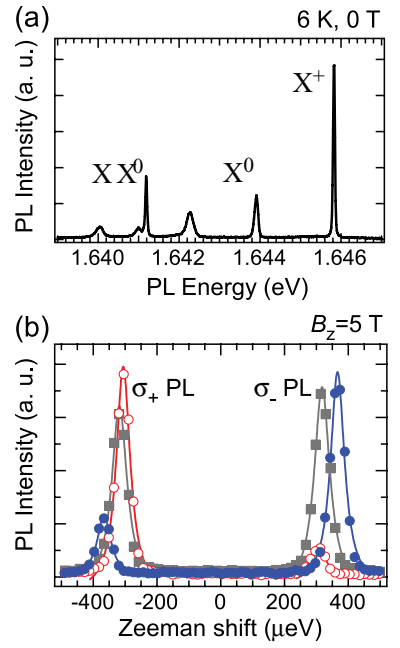

(c)

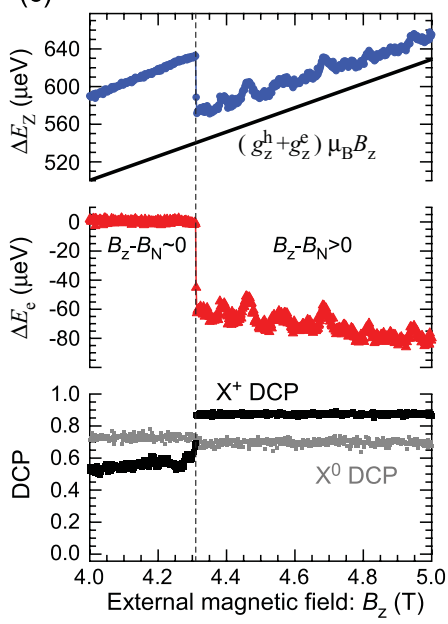

FIG. 1. (Color online) (a) PL spectra from the target single QD at zero magnetic field. (b) PL spectra of $\mathrm{X}^{+}$state at $B_{\mathrm{z}}=5 \mathrm{~T}$ with linearly (gray squares) and circularly $\left(\sigma_{+} / \sigma_{-}\right.$: open/solid circles) polarized excitations. The $\sigma_{-(+)}$PL component is positioned at the higher (lower) energy side. The difference between the Zeeman splitting $\Delta E_{\mathrm{Z}}$ for the circularly and linearly polarized excitations is defined as the Overhauser shift $\left(\Delta E_{\mathrm{OHS}}\right)$, and it is evaluated as $\Delta E_{\mathrm{OHS}}=98 \mu \mathrm{eV}(-21 \mu \mathrm{eV})$ for $\sigma_{-}\left(\sigma_{+}\right)$excitation. (c) $B_{\mathrm{z}}$ dependencies of $\Delta E_{\mathrm{Z}}$ of the $\mathrm{X}^{+} \mathrm{PL}$ line (upper panel), the energy splitting of the electronic level $\Delta E_{\mathrm{e}}$ (middle panel), and the DCPs of $\mathrm{X}^{+}$and $\mathrm{X}^{0}$ (denoted by the black and gray symbols in lower panel) for $\sigma_{-}$excitation. The solid line in the upper panel is the calculated $\Delta E_{\mathrm{Z}}$ at $B_{\mathrm{N}}=0$.

Zeeman interaction of the spins with the (effective) magnetic fields. Under this condition, $\Delta E_{\mathrm{Z}}$ can be expressed as follows: $\Delta E_{\mathrm{Z}}=g_{\mathrm{z}}^{\mathrm{h}} \mu_{\mathrm{B}}\left|\boldsymbol{B}_{\mathrm{Z}}\right|+g_{\mathrm{z}}^{\mathrm{e}} \mu_{\mathrm{B}}\left|\boldsymbol{B}_{\mathrm{Z}}+\boldsymbol{B}_{\mathrm{N}}\right|$, where $g_{\mathrm{z}}^{\text {e(h) }}$ denotes the electron (hole) $g$ factor in the growth direction and $\boldsymbol{B}_{\mathrm{N}}$ denotes the Overhauser field. Since the hole spin has a low probability of existence at the nucleus site, the effect of $B_{\mathrm{N}}$ on the hole spin could be neglected except for a special case. ${ }^{23}$ Under a large $B_{\mathrm{Z}}$ of a few teslas, $B_{\mathrm{N}}$ manifests itself as an OHS defined as $\Delta E_{\mathrm{OHS}}=g_{\mathrm{z}}^{\mathrm{e}} \mu_{\mathrm{B}} B_{\mathrm{N}}$. Since $B_{\mathrm{N}}$ is essentially zero for the linearly polarized excitation, OHS is deduced from the difference between $\Delta E_{\mathrm{Z}}$ for the circularly and linearly polarized excitations, and it is evaluated in Fig. 1(b) as $\Delta E_{\mathrm{OHS}}=98$ $\mu \mathrm{eV}(-21 \mu \mathrm{eV})$ with $\sigma_{-}\left(\sigma_{+}\right)$excitation. As per our definition, the $\sigma_{-}\left(\sigma_{+}\right)$excitation generates $B_{\mathrm{N}}$ in the opposite (same) direction to $B_{\mathrm{Z}}$, and it induces an apparent increase (decrease) in $\Delta E_{\mathrm{Z}}$ because of the relation $g_{\mathrm{z}}^{\mathrm{e}} g_{\mathrm{z}}^{\mathrm{h}}<0$. The large difference of the OHS observed for $\sigma_{+}$and $\sigma_{-}$excitations is due to the bistable response of NSP. ${ }^{8}$ In the case of $\sigma_{-}$excitation, the energy mismatch in e-n spin flip-flop process deceases by increasing $B_{\mathrm{N}}$, which induces the bistability of NSP, and the large OHS in the upper NSP branch was achieved. On the other hand, the increasing energy mismatch for $\sigma_{+}$excitation prevents from occurring the bistable response, and as a result, only a small OHS was created.

Hereafter, we focus on the $\sigma_{-}$case where the compensation of $B_{\mathrm{Z}}$ via $B_{\mathrm{N}}$ is achieved; consequently, the bistabilities of NSP have been observed for external parameters such as the excitation power, ${ }^{8}$ excitation polarization, ${ }^{7}$ and external magnetic field. ${ }^{9}$ 
Figure 1(c) summarizes the effects of NSP on the $\mathrm{X}^{+} \mathrm{PL}$ observed in the $B_{\mathrm{z}}$ dependence measurement for $\sigma_{-}$excitation. In the experiment, the excitation power was fixed, and the external field was swept from 4.0 T to 5.0 T with a sweeping rate of $0.11 \mathrm{~T} / \mathrm{min}$. The symbols and the solid line in the upper panel indicate the observed $\Delta E_{\mathrm{Z}}$ and the calculated $\Delta E_{\mathrm{Z}}$ under the condition when $B_{\mathrm{N}}=0$. The difference from the zero $B_{\mathrm{N}}$ line is the OHS at each $B_{\mathrm{z}}$. As can be clearly observed, an abrupt decrease in $\Delta E_{\mathrm{Z}}$ was observed at $B_{\mathrm{Z}}=4.31 \mathrm{~T}$ owing to the bistable nature of NSP. In order to measure the degree of $B_{\mathrm{Z}}$ compensation via $B_{\mathrm{N}}$, we introduce the effective magnetic field as experienced by e-spin: $\boldsymbol{B}_{\text {eff }}\left(=\boldsymbol{B}_{\mathrm{z}}+\boldsymbol{B}_{\mathrm{N}}\right)$. By using the previously obtained values of $g_{\mathrm{z}}^{\mathrm{h}}=+2.54$ and $g_{\mathrm{z}}^{\mathrm{e}}=-0.37,{ }^{5}$ we deduced the electronic splitting energy $\Delta E_{\mathrm{e}}=g_{\mathrm{z}}^{\mathrm{e}} \mu_{\mathrm{B}} B_{\text {eff }}$, as shown in the middle panel of Fig. 1(c). In the region where $B_{\mathrm{z}}<4.31 \mathrm{~T}$, the absolute value of $\Delta E_{\mathrm{e}}$ nearly reduces to zero, and the Overhauser field compensates for the external field within the homogeneous broadening of the e-n spin system. With increasing $B_{\mathrm{z}}$, the magnitude of $B_{\mathrm{N}}$ shows a clear reduction and $\left|\Delta E_{\mathrm{e}}\right|$ increases abruptly.

Here, we focus on the DCP of the $\mathrm{X}^{+}$PL [the lower panel of Fig. 1(c)]. In this work, the DCP is defined as $\left(I^{-}-I^{+}\right) /\left(I^{-}+\right.$ $\left.I^{+}\right)\left(I^{+(-)}\right.$denotes the integrated PL intensity of the $\sigma_{+(-)}$ component). It is noteworthy that the DCP of $\mathrm{X}^{+}$PL shows a clear jump from $\sim 0.6$ to $\sim 0.9$; this transition synchronizes with the decrease in $\Delta E_{\mathrm{e}}$. As mentioned above, the DCP of $\mathrm{X}^{+}$is essentially determined solely by the e-spin polarization $\left\langle S_{\mathrm{z}}\right\rangle$, and it can be expressed as DCP $=2\left\langle S_{\mathrm{z}}\right\rangle$. Accordingly, a high (low) value of DCP indicates a small (large) reduction in e-spin polarization (i.e., e-spin relaxation). Since the e-spin relaxation rate depends on $\left|\Delta E_{\mathrm{e}}\right|$, the change in the $\mathrm{X}^{+} \mathrm{DCP}$ presents the possibility of a direct measurements of the electron and nuclear spin polarizations in a coupled e-n system.

It is noteworthy that the DCPs observed in the other charge states show different behaviors. The OHSs observed in the $\mathrm{X}^{0}$ and $\mathrm{XX}^{0}$ PLs show changes similar to that observed in $\mathrm{X}^{+}$; this is one of the pieces of evidence that these PL lines originate from the same QD. ${ }^{24}$ In contrast, the tendencies of DCP are quite different for these other exciton complex peaks. The $\mathrm{X}^{0}$ DCP stays almost constant $(\sim 0.7)$, thereby signifying insensitivity to or independence from $\Delta E_{\mathrm{e}}$, as shown in the lower panel of Fig. 1(c) (denoted by the gray symbols). This can partially be attributed to the contribution of the unpolarized $\mathrm{X}^{0}$ supplied from the $\mathrm{XX}^{0} . \mathrm{XX}^{0}$ decays to $\mathrm{X}^{0}$ by emitting $\sigma_{+}$ and $\sigma_{-}$photons with identical probabilities, and therefore $\mathrm{XX}^{0}$ DCP is basically zero (not shown here). The DCP of $\mathrm{X}^{0}$ is approximately calculated as $[(n+n / 4)-n / 4] /[(n+n / 4)+$ $n / 4] \sim 0.67$, if QDs are excited under the power at which $n$ electron-hole pairs $\left(\mathrm{X}^{0}\right)$ are generated in each QD (in this case, $\mathrm{XX}^{0} / \mathrm{X}^{0}=1 / 2$ according to Poisson statics). Further studies in this direction require a close examination of the dynamics of the whole $\mathrm{X}^{0}$ states which include not only the bright exciton but the dark exciton states.

\section{B. Experimental estimation of nuclear field fluctuation and electron spin relaxation time}

Here, we estimate the key quantities $\left(\Delta B_{\mathrm{N}}\right.$ and $\left.T_{\Delta}\right)$ from the experimental data. Based on the standard theory, ${ }^{11,14}$ in the initial stage of the e-n spin dynamics, the Overhauser field

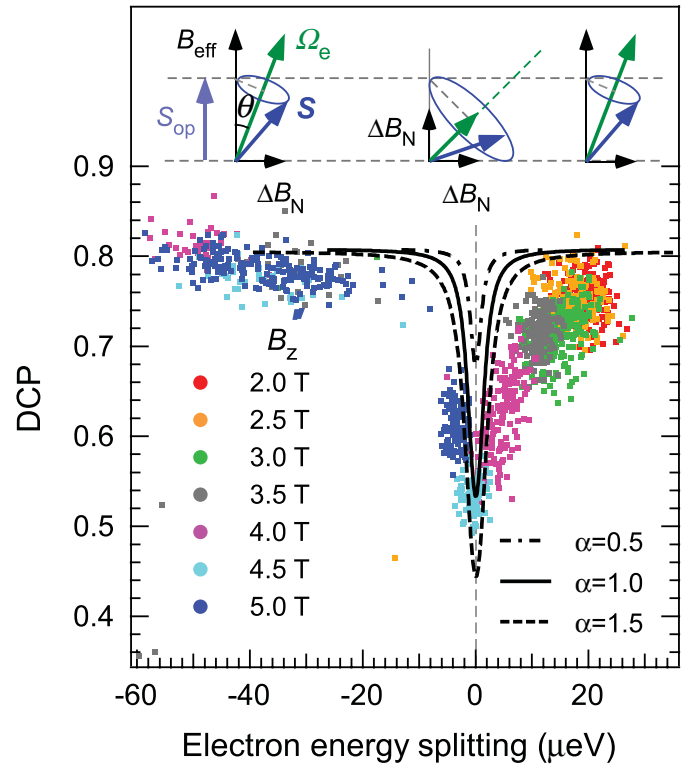

FIG. 2. (Color online) $\mathrm{X}^{+}$DCPs as a function of the electronic energy splitting at the different external field values. Symbols and colors indicate the experimental data and corresponding $B_{z}$. Each of the data values was obtained from the time-resolved measurements of OHS and DCP like Fig. 3(b). The absence of the data points around $\Delta E_{\mathrm{e}} \sim-15 \mu \mathrm{eV}$ is attributed to the abrupt changes in OHS and DCP. The curves are the calculated results obtained for $\alpha\left(=\tau_{\mathrm{R}} / T_{\Delta}\right)=0.5$, 1.0 , and 1.5, respectively. Inset is the schematic of the precession of the e-spin $\boldsymbol{S}$ around the torque vector $\boldsymbol{\Omega}_{\mathrm{e}}$. The $\boldsymbol{\Omega}_{\mathrm{e}}$ is composed of the effective magnetic field $B_{\text {eff }}$ as the $z$ component and an isotropic fluctuation of Overhauser field $\Delta B_{\mathrm{N}}$.

$\boldsymbol{B}_{\mathrm{N}}$ can be considered to be frozen because its correlation time, which is determined by the dipole-dipole interaction between nuclei, is in the microsecond range at least and is quite longer than the recombination time $\tau_{\mathrm{R}} \sim 0.75 \mathrm{~ns}$ obtained by other independent time-resolved measurements. ${ }^{18}$ In this situation, the QD-confined e-spin just precesses coherently around the torque vector $\boldsymbol{\Omega}_{\mathrm{e}}$. However, due to the random variable of the transverse component of Overhauser field $\Delta B_{\mathrm{N}}$, the e-spin polarization decreases from its initial value within the characteristic time $T_{\Delta}$. Assuming that the orientation of $\Delta B_{\mathrm{N}}$ is randomly distributed over the accumulation time of the CCD detector $(0.1-1 \mathrm{~s})$, the DCP of time-integrated $\mathrm{X}^{+}$ $\mathrm{PL}$ is given by ${ }^{11,25}$

$$
\mathcal{P}_{\mathrm{c}}=\frac{2}{\tau_{\mathrm{R}}} \int\left\langle S_{\mathrm{Z}}(t)\right\rangle \exp \left(-t / \tau_{\mathrm{R}}\right) d t,
$$

where $\left\langle S_{\mathrm{Z}}(t)\right\rangle$ is the temporal evolution of the e-spin polarization averaged over the distribution of $\Delta B_{\mathrm{N}}$.

As discussed by Merkulov et al. in detail, ${ }^{11}\left\langle S_{\mathrm{Z}}(t)\right\rangle$ is given as a function of $\Delta B_{\mathrm{N}}$, and its behavior is qualitatively explained by the evolution of the angle between $z$ axis and $\boldsymbol{\Omega}_{\mathrm{e}}$ (shown in the inset of Fig. 2). Here, $\boldsymbol{\Omega}_{\mathrm{e}}$ can be expressed as follows: $\boldsymbol{\Omega}_{\mathrm{e}}=g_{\mathrm{z}}^{\mathrm{e}} \mu_{\mathrm{B}} \boldsymbol{B}_{\text {eff }} / \hbar$, where an isotropic fluctuation of the Overhauser field $\Delta B_{\mathrm{N}}$ is included. While the transverse component of $\boldsymbol{\Omega}_{\mathrm{e}}$, which is denoted by $\Omega_{\mathrm{e}}^{\perp}$ and is determined by the inherent quantity $\Delta B_{\mathrm{N}}$, could not be changed by the external parameters, the longitudinal component of $\boldsymbol{\Omega}_{\mathrm{e}}$ ( similarly denoted by $\Omega_{\mathrm{e}}^{\|}$) is adjustable via the external and 
(a)

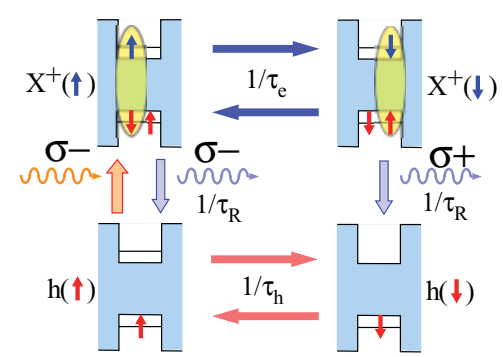

(b)

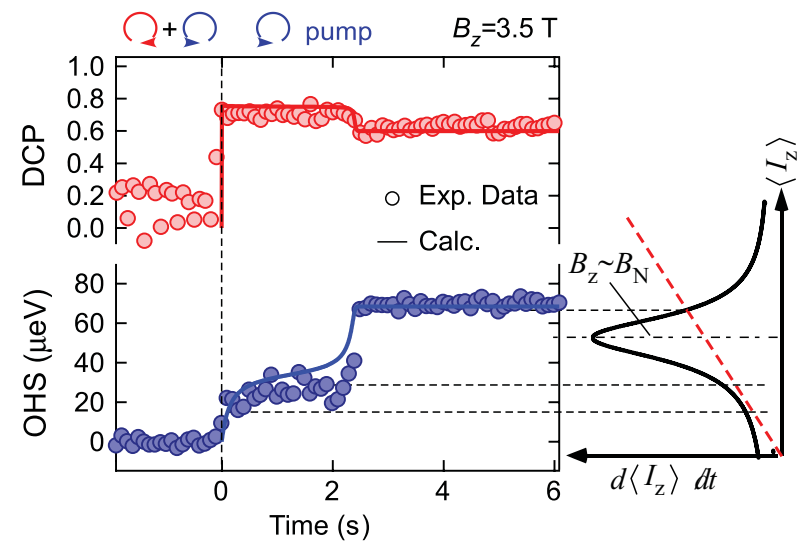

FIG. 3. (Color online) (a) Current dynamics model of e-spin system including $\mathrm{X}^{+}$and the single-hole states, and the corresponding PL polarizations. (b) Transient evolutions of DCP and OHS at $B_{\mathrm{z}}=3.5 \mathrm{~T}$. The excitation polarizations are depicted in the upper side of the panel. The solid curves are the calculated results in the coupled e-n system, and they were able to reproduce all the behaviors of the experimental results. Right inset indicates a schematic of the n-spin polarization (black solid curve) and depolarization (red dashed line) terms of Eq. (2) for an explanation of the transient OHS.

the Overhauser fields. In a large effective field $\left(B_{\text {eff }} \gg \Delta B_{\mathrm{N}}\right)$, the torque vector is nearly along the $z$ axis, and $\left\langle S_{z}(t)\right\rangle$ stays at the value $S_{\mathrm{op}}$, where $S_{\mathrm{op}}$ is the initial e-spin polarization injected into the QD ground state. On the other hand, in a weak effective field ( $\left.B_{\text {eff }} \sim \Delta B_{\mathrm{N}}\right), \boldsymbol{\Omega}_{\mathrm{e}}$ is dominantly determined by $\Delta B_{\mathrm{N}}$, and $\left\langle S_{\mathrm{z}}(t)\right\rangle$ in the long time limit converges to $S_{\mathrm{op}} / 3$. Therefore, $\mathcal{P}_{\mathrm{c}}$ is expected to show a dip structure at the point of $\Delta E_{\mathrm{e}}=g^{\mathrm{e}} \mu_{\mathrm{B}} B_{\mathrm{eff}} \sim 0$, and taking advantage of this feature, the invariant $\Delta B_{\mathrm{N}}$ can be evaluated from the DCP and the corresponding $\Delta E_{\mathrm{e}}$ measurements.

Figure 2 shows the DCPs as a function of $\Delta E_{\mathrm{e}}$ at different values of external field strength. For the construction of Fig. 2, the data points obtained from the time-resolved measurements of DCP and OHS of $\mathrm{X}^{+}$PL [e.g., Fig. 3(b)] were used; the deduced OHS were converted to $\Delta E_{\mathrm{e}}$. Many sets of DCP and the corresponding $\Delta E_{\mathrm{e}}$ under different magnetic fields ( $2 \mathrm{~T}$ $\leqslant B_{\mathrm{Z}} \leqslant 5 \mathrm{~T}$ ) were plotted on Fig. 2. As clearly shown, a definite dip is observed at $\Delta E_{\mathrm{e}} \simeq 0$. It should be noted that the $\mathrm{X}^{+}$DCPs at the different $B_{\mathrm{z}}$ values depict a unique curve and this fact justifies the assumption that $\Delta B_{\mathrm{N}}$, which is the key quantity to determine $\left\langle S_{\mathrm{Z}}(t)\right\rangle$ and the resulting DCP, is independent of $B_{\mathrm{z}}$.

Here, we compare the experimentally obtained results and the calculations. For convenience, $\mathcal{P}_{\mathrm{c}}$ is expressed as a function of the dimensionless parameters $\alpha=\tau_{\mathrm{R}} / T_{\Delta}$ and $\beta=B_{\text {eff }} / \Delta B_{\mathrm{N}}$. Then, Eq. (1) can be written as $\mathcal{P}_{\mathrm{c}}(\alpha, \beta)=$ $\frac{2}{\alpha} \int_{0}^{\infty}\left\langle S_{\mathrm{z}}(\beta, \gamma)\right\rangle \exp (-\gamma / \alpha) d \gamma$, where $\gamma=t / T_{\Delta}$. For the $B_{\text {eff }}$ dependence with a given $\alpha$, the $\mathcal{P}_{\mathrm{c}}$ was calculated by changing the variable $\beta$. The curves in Fig. 2 were the calculated results obtained for $\alpha=0.5,1.0$, and 1.5. They were able to reproduce the dip structure at $\Delta E_{\mathrm{e}} \simeq 0$, and their behaviors were understood intuitively by the above-mentioned evolution of the angle between $z$ axis and $\boldsymbol{\Omega}_{\mathrm{e}}$, i.e., $\theta=\tan ^{-1}\left(\Delta B_{\mathrm{N}} / B_{\text {eff }}\right)$. This implies that the effect of $\Omega_{\mathrm{e}}^{\perp}$ could be screened out with the strong $B_{\text {eff }}$. By increasing the parameter $\alpha$, the amplitude and the width of the dip structure in calculations increase clearly; this is due to the large e-spin relaxation rate (approximately proportional to $T_{\Delta}^{-1}$ ) for a fixed $\tau_{\mathrm{R}}$. In adopting $\alpha=1$ for the best fit of the observed DCP variation, the e-spin relaxation time $T_{\Delta}$ was estimated to be $\tau_{\mathrm{R}}(\sim 0.75 \mathrm{~ns})$. Additionally, $\Delta B_{\mathrm{N}}$ can be evaluated to be $\sim 40 \mathrm{mT}$ from the relation of $\Delta B_{\mathrm{N}}=\hbar /\left(g^{\mathrm{e}} \mu_{\mathrm{B}} T_{\Delta}\right)$. By the way, the fluctuation of the Overhauser field can be estimated theoretically as $\Delta B_{\mathrm{N}} \cong A I /\left(\sqrt{N} g^{\mathrm{e}} \mu_{\mathrm{B}}\right)$ ( $A$ : the hyperfine coupling constant, $I$ : the nuclear spin moment $){ }^{26}$ By using the typical values for an $\mathrm{In}_{0.75} \mathrm{Al}_{0.25} \mathrm{As} \mathrm{QD}\left(g^{\mathrm{e}}, A, N, I\right)=(-0.37,50 \mu \mathrm{eV}$, $\left.3 \times 10^{4}, 11 / 4\right)$, we can roughly deduce $\Delta B_{\mathrm{N}} \approx 40 \mathrm{mT}$ and $T_{\Delta} \approx 1 \mathrm{~ns}$. These values agree well with the calculated ones with $\alpha=1$. The observed $\Delta B_{\mathrm{N}}$ is comparable to the values reported in InAs $\mathrm{QDs}^{25,27}(\sim 30 \mathrm{mT})$ and in InP $\mathrm{QDs}^{28}$ $(\sim 15 \mathrm{mT})$.

Although the calculations obtained in the framework of the standard e-spin relaxation theory in QDs can qualitatively explain the behavior of the measured data very well, the dip width obtained from the measurements is approximately three times wider than the calculated one. It should be noted that the accuracy of the parameters $\left(\tau_{\mathrm{R}}\right.$ and $\left.g^{\mathrm{e}}\right)$ used in the calculation is inadequate to explain the difference between the observed data and the theoretical prediction. As mentioned before, $\tau_{\mathrm{R}}$ affects the DCP curve through the dimensionless parameter $\alpha=\tau_{\mathrm{R}} / T_{\Delta}$; therefore, the calculated curve which gives the best fit of the observations $(\alpha=1)$ is independent from the value of $\tau_{\mathrm{R}}$. Furthermore, the electronic $g$ factor of the target QD was successfully evaluated with high precision $\left(g^{\mathrm{e}}=-0.37 \pm 0.02\right),{ }^{5}$ and within this accuracy, the observed wider DCP curve with an appropriate amplitude could not be reproduced. This suggests that the extrinsic e-spin relaxation mechanism may affect the observation. In this point, further experimental and theoretical studies are required. In addition, the estimated $T_{\Delta}$ is in good agreement with the exciton spin relaxation time obtained in the photon correlation, ${ }^{19}$ Hanle-like measurements, ${ }^{29}$ and 3-pulse four wave mixing measurements ${ }^{30}$ of the InAlAs QDs.

In this section, the fluctuation of the Overhauser field and the e-spin relaxation time, two key quantities in e-n spin dynamics, were evaluated from the experimental data. The saturation of DCP at large $B_{\text {eff }}$ region indicates that the $B_{\text {eff }}$ is able to screen the effect of the transverse component of $\Delta B_{\mathrm{N}}$. Additionally, the experimental data except for the dip width could be explained well with the calculations based on the standard spin relaxation theory for the QD-confined electron in the context of the typical values of InAlAs QDs. The obtained spin relaxation time can explain reasonably the exciton spin relaxation time obtained in other measurements for resonant and nonresonant excitations under no external magnetic fields. 


\section{Dynamics model of coupled electron-nuclear spin system}

Finally, we test the validity of the dynamics model of the coupled e-n spin system. The temporal evolution of the mean NSP $\left\langle I_{z}\right\rangle$ is described by the following rate equation: ${ }^{31}$

$$
\frac{d\left\langle I_{\mathrm{z}}\right\rangle}{d t}=\frac{1}{T_{\mathrm{NF}}}\left[Q\left(\left\langle S_{\mathrm{z}}\right\rangle-S_{0}\right)-\left\langle I_{\mathrm{z}}\right\rangle\right]-\frac{1}{T_{\mathrm{ND}}}\left\langle I_{\mathrm{z}}\right\rangle,
$$

where $S_{0}$ denotes the thermal e-spin polarization, $1 / T_{\mathrm{NF}}$ and $1 / T_{\mathrm{ND}}$ denote the $\mathrm{n}$-spin polarization and depolarization rates, ${ }^{32}$ respectively, and $Q=I(I+1) /[S(S+1)]$ denotes the momentum conversion coefficient from the e-spin to n-spin system. The first term on the right-hand side of Eq. (2) represents the n-spin polarization, and the second term represents the $n$-spin depolarization. The $n$-spin polarization rate is given as ${ }^{15}$

$$
\frac{1}{T_{\mathrm{NF}}}=\left[f_{\mathrm{e}} \tau_{\mathrm{c}}\left(\frac{A}{N \hbar}\right)^{2}\right] /\left[1+\left(\frac{\tau_{\mathrm{c}}}{\hbar}\right)^{2}\left(\left|g_{\mathrm{z}}^{\mathrm{e}} \mu_{\mathrm{B}} B_{\mathrm{z}}\right| \pm\left|A\left\langle I_{\mathrm{z}}\right\rangle\right|\right)^{2}\right],
$$

where $f_{\mathrm{e}}$ denotes the factor of finite electron occupancy in the $\mathrm{QD}\left(f_{\mathrm{e}} \leqslant 1\right)$ and $\tau_{\mathrm{c}}$ is the permanence of the flip-flop term of the hyperfine interaction. ${ }^{7}$ The sign before $\left|A\left\langle I_{\mathrm{Z}}\right\rangle\right|$ is determined by the sign of the Overhauser field $B_{\mathrm{N}}\left(=A\left\langle I_{\mathrm{z}}\right\rangle / g_{\mathrm{z}}^{\mathrm{e}} \mu_{\mathrm{B}}\right)$. As mentioned in the previous section, we focus on the $\sigma_{-}$ excitation case where $B_{\mathrm{N}}$ is created in the opposite direction to $B_{\mathrm{z}}$; therefore the negative sign was adopted in the following calculations. This equation represents a Lorentzian shape with a full width at half maximum of $2 \hbar / \tau_{\mathrm{c}}$ in the electronic energy splitting as shown in the right inset of Fig. 3(b). Since the NSP formation rate is very sensitive to the degree of energy mismatch in the e-n flip-flop process, which is determined by the splitting and the broadening of the corresponding e-n levels, $\tau_{\mathrm{c}}$ has a crucial influence on the NSP dynamics. In the calculations to reproduce the observations, $\tau_{\mathrm{c}}$ of several picoseconds were used,,${ }^{6,7,9}$ and the value is in the same order of magnitude as the $\mathrm{X}^{+}$decoherence time ( $\sim 3 \mathrm{ps)}$ that was measured by Fourier spectroscopy of the same single InAlAs QD. ${ }^{33}$ Although Eq. (2), with constant values of the averaged e-spin polarization $\left\langle S_{\mathrm{z}}\right\rangle$, has explained the observed dependence of OHS qualitatively in previous studies, the actual $\left\langle S_{\mathrm{Z}}\right\rangle$ in the dynamics is expected to change along with the evolution of $\left\langle I_{\mathrm{z}}\right\rangle$.

In our model calculation, $\left\langle S_{z}\right\rangle$, which drags the randomly oriented n-spin ensemble to the highly polarized state, is determined by the dynamics in the following four states, as shown in Fig. 3(a): $\mathrm{X}^{+}$with the spin-up/down electron $\left(n_{\uparrow}\right.$ and $n_{\downarrow}$ : the populations of the corresponding states), and the spin-up/down single-hole states (similarly denoted by $n_{\Uparrow}$ and $\left.n_{\Downarrow}\right)$, and $\left\langle S_{\mathrm{Z}}(t)\right\rangle$ is given as $\left[n_{\uparrow}(t)-n_{\downarrow}(t)\right] /\left\{2\left[n_{\uparrow}(t)+n_{\downarrow}(t)\right]\right\}$. These four states are connected with the rates of the optical pumping with $\sigma_{-}$light, the radiative recombination $\left(1 / \tau_{\mathrm{R}}\right)$, and the spin flips of electron and hole $\left(1 / \tau_{\mathrm{e}}\right.$ and $\left.1 / \tau_{\mathrm{h}}\right)$. The initial populations at the time of origin were given as $n_{\uparrow}(0)=n_{\downarrow}(0)=0$ and $n_{\Uparrow}(0)=n_{\Downarrow}(0)=0.5$, respectively. The population of the spin-up single-hole state decreases due to the optical pumping with the $\sigma_{-}$light, and the population of the $\mathrm{X}^{+}$with the spin-up electron increases $\left(n_{\Uparrow} \rightarrow n_{\uparrow}\right)$. The optically pumped population of spin-up $\mathrm{X}^{+}$ is partially transferred to the one of spin-down $\mathrm{X}^{+}\left(n_{\uparrow} \rightarrow n_{\downarrow}\right)$ via the e-spin flip process. Note that (i) the e-spin flip rate $1 / \tau_{\mathrm{e}}$ is approximately written as a Lorentzian function in the electronic energy splitting and its width and amplitude are deduced from the calculation shown in Fig. 2, and (ii) the reverse process, that is, the population transfer from the spin-down $\mathrm{X}^{+}$to spin-up $\mathrm{X}^{+}\left(n_{\downarrow} \rightarrow n_{\uparrow}\right)$, would occur with the same rate. After the duration of $\tau_{R}$, the populations of $\mathrm{X}^{+}$ states are transferred to the ones of single-hole spins due to the radiative recombination $\left(n_{\uparrow(\downarrow)} \rightarrow n_{\Uparrow(\downarrow)}\right)$. The population of the spin-up (spin-down) single hole is transferred to the single-hole state with the opposite spin $\left(n_{\Uparrow} \leftrightarrow n_{\Downarrow}\right)$ with the rate of $1 / \tau_{\mathrm{h}}$. The rate equations for the populations of these four states and for the NSP were solved numerically by using the obtained $T_{\Delta}$, and the temporal evolutions of OHS $\left(=A\left\langle I_{\mathrm{z}}\right\rangle\right)$ and $\mathrm{X}^{+}$DCP were calculated.

The direct observation of the temporal evolutions of OHS and DCP can provide a better understanding of the e- $n$ spin dynamics. Typical transients obtained from the target $\mathrm{X}^{+} \mathrm{PL}$ at $B_{\mathrm{z}}=3.5 \mathrm{~T}$ are shown in Fig. 3(b). In order to set the initial NSP to zero, the excitation polarization before the time of origin was modulated between $\sigma_{+}$and $\sigma_{-}$, with a frequency of $10 \mathrm{~Hz}$. In this time region, the DCP is noisy around zero due to the nonsynchronous detection of the PL signals with the modulation period of the excitation polarization.

The temporal evolution of OHS for $\sigma_{-}$excitation, which is described by Eq. (2), can be understood schematically by the difference between the Lorentzian-shaped polarization (a black solid curve) and the depolarization (a red dashed straight line), as shown in the right inset of Fig. 3(b). After switching to $\sigma_{-}$excitation, OHS increases gradually in the region of small difference and increases explosively around the peak of the n-spin polarization rate. Finally, the steady-state value of OHS goes to the balance points between polarization and depolarization terms. Under this experimental condition, the OHS jumps clearly to the saturated value within $3 \mathrm{~s}$, and $B_{\mathrm{z}}$ compensation via $B_{\mathrm{N}}$ is achieved within the homogeneous broadening of the n-spin polarization rate. At the exact moment of the abrupt increase in the OHS, the DCP of the $\mathrm{X}^{+} \mathrm{PL}$ drops suddenly from 0.8 to 0.7 . The solid curves are the calculated results obtained from the above-mentioned dynamics model. In the calculation, the key parameters $\left(\Delta B_{\mathrm{N}}\right.$ and $\left.T_{\Delta}\right)$ were in the same order as the experimentally evaluated values reported in a previous section of this paper. The fact that the calculation could reproduce the observed DCP as well as OHS shows the validity of our dynamics model.

\section{CONCLUSION}

In conclusion, we investigated the spin dynamics of the coupled electron-nuclear spin system in a single InAlAs QD. The DCP of $\mathrm{X}^{+}$PL, which is basically determined by e-spin polarization, showed synchronized changes with the electronic energy splitting, and this fact offers the possibility of NSP probing via $\mathrm{X}^{+}$DCP in a QD structure. By taking advantage of this feature, the fluctuation of Overhauser field and the e-spin relaxation time, which play a crucial role in spin dynamics, were evaluated as $\Delta B_{\mathrm{N}} \sim 40 \mathrm{mT}$ and $T_{\Delta} \sim 0.75 \mathrm{~ns}$, respectively. The experimentally obtained values agree well with the results obtained by calculations. Additionally, the experimental data except for its dip width could be explained 
well with the calculations based on the standard spin relaxation theory for the QD-confined electron in context of the typical values of InAlAs QDs. The spin dynamics model used in this study successfully reproduces the observations of DCP as well as the OHS, and we believe that the model can significantly contribute to the understanding of e-n spin dynamics. *r-kaji@eng.hokudai.ac.jp

${ }^{1}$ J. M. Taylor, C. M. Marcus, and M. D. Lukin, Phys. Rev. Lett. 90, 206803 (2003).

${ }^{2}$ S. Muto, S. Adachi, T. Yokoi, H. Sasakura, and I. Suemune, Appl. Phys. Lett. 87, 112506 (2005).

${ }^{3}$ D. Gammon, Al. L. Efros, T. A. Kennedy, M. Rosen, D. S. Katzer, D. Park, S. W. Brown, V. L. Korenev, and I. A. Merkulov, Phys. Rev. Lett. 86, 5176 (2001).

${ }^{4}$ T. Yokoi, S. Adachi, H. Sasakura, S. Muto, H. Z. Song, T. Usuki, and S. Hirose, Phys. Rev. B 71, 041307(R) (2005).

${ }^{5}$ R. Kaji, S. Adachi, H. Sasakura, and S. Muto, Appl. Phys. Lett. 91, 261904 (2007).

${ }^{6}$ R. Kaji, S. Adachi, H. Sasakura, and S. Muto, Phys. Rev. B 77, 115345 (2008).

${ }^{7}$ P.-F. Braun, B. Urbaszek, T. Amand, X. Marie, O. Krebs, B. Eble, A. Lemaître, and P. Voisin, Phys. Rev. B 74, 245306 (2006).

${ }^{8}$ A. I. Tartakovskii, T. Wright, A. Russell, V. I. Fal'ko, A. B. Van'kov, J. Skiba-Szymanska, I. Drouzas, R. S. Kolodka, M. S. Skolnick, P. W. Fry, A. Tahraoui, H.-Y. Liu, and M. Hopkinson, Phys. Rev. Lett. 98, 026806 (2007).

${ }^{9}$ P. Maletinsky, C. W. Lai, A. Badolato, and A. Imamoglu, Phys. Rev. B 75, 035409 (2007).

${ }^{10}$ E. A. Chekhovich, M. N. Makhonin, J. Skiba-Szymanska, A. B. Krysa, V. D. Kulakovskii, M. S. Skolnick, and A. I. Tartakovskii, Phys. Rev. B 81, 245308 (2010).

${ }^{11}$ I. A. Merkulov, Al. L. Efros, and M. Rosen, Phys. Rev. B 65, 205309 (2002).

${ }^{12}$ I. A. Merkulov, G. Alvarez, D. R. Yakovlev, and T. C. Schulthess, Phys. Rev. B 81, 115107 (2010).

${ }^{13}$ P.-F. Braun, X. Marie, L. Lombez, B. Urbaszek, T. Amand, P. Renucci, V. K. Kalevich, K. V. Kavokin, O. Krebs, P. Voisin, and Y. Masumoto, Phys. Rev. Lett. 94, 116601 (2005).

${ }^{14}$ Spin Physics in Semiconductors, Springer Series in Solid-State Sciences Vol. 157, Chaps. 1 and 11, edited by M. I. Dyakonov (Springer, Berlin, 2008).

${ }^{15}$ Optical Orientation, Modern Problems in Condensed Matter Sciences Vol. 8, Chaps. 2 and 5, edited by F. Meier and B. Zakharchenya (North-Holland, NewYork, 1984).
${ }^{16}$ H. Sasakura, S. Adachi, S. Muto, H. Z. Song, T. Miyazawa, and Y. Nakata, Physica E 21, 511 (2004).

${ }^{17}$ H. Sasakura, S. Adachi, S. Muto, H. Z. Song, T. Miyazawa, and T. Usuki, Jpn., J. Appl. Phys. 43, 2110 (2004).

${ }^{18}$ R. Kaji, doctoral thesis, Hokkaido University, 2011.

${ }^{19}$ H. Kumano, S. Kimura, M. Endo, H. Sasakura, S. Adachi, S. Muto, and I. Suemune, J. Nanoelectron. Optoelectron. 1, 39 (2006).

${ }^{20}$ R. Kaji, K. Yamada, H. Sasakura, and S. Adachi, Phys. Status Solidi B 245, 2662 (2008).

${ }^{21}$ S. Adachi, H. Sasakura, N. Yatsu, R. Kaji, K. Yamada, S. Muto, H. Kumano, and I. Suemune, Phys. Status Solidi C 5, 351 (2008).

${ }^{22}$ T. Tsuchiya, Physica E 7, 470 (2000).

${ }^{23}$ B. Eble, C. Testelin, P. Desfonds, F. Bernardot, A. Balocchi, T. Amand, A. Miard, A. Lemaître, X. Marie, and M. Chamarro, Phys. Rev. Lett. 102, 146601 (2009).

${ }^{24}$ H. Sasakura, R. Kaji, S. Adachi, and S. Muto, Appl. Phys. Lett. 92, 041915 (2008)

${ }^{25}$ O. Krebs, B. Eble, A. Lemaître, P. Voisin, B. Urbaszek, T. Amand, and X. Marie, C. R. Phys. 9, 874 (2008).

${ }^{26} \mathrm{P}$. Maletinsky, Optical Orientation of Nuclear Spins in an Individual Quantum Dot, Chap. 3 (Südwestdeutcher Verlag für Hochschulschriften, 2008).

${ }^{27}$ O. Krebs, B. Eble, A. Lemaître, B. Urbaszek, K. Kowalik, A. Kudelski, X. Marie, T. Amand, and P. Voisin, Phys. Status. Solidi. A 204, 202 (2007).

${ }^{28}$ B. Pal, S. Y. Verbin, I. V. Ignatiev, M. Ikezawa, and Y. Masumoto, Phys. Rev. B 75, 125322 (2007).

${ }^{29}$ R. Kaji, S. Adachi, T. Shindo, and S. Muto, Phys. Rev. B 80, 235334 (2009).

${ }^{30}$ T. Watanuki, S. Adachi, H. Sasakura, and S. Muto, Appl. Phys. Lett. 86, 063114 (2005).

${ }^{31}$ A. Abragam, The Principles of Nuclear Magnetism (Clarendon, Oxford, 1961).

${ }^{32}$ For simplicity, the magnetic field dependence of $1 / T_{\mathrm{ND}}$ is ignored here and it is not necessary to introduce this dependence at the present stage.

${ }^{33}$ S. Adachi, N. Yatsu, R. Kaji, and S. Muto, Appl. Phys. Lett. 91, 161910 (2007) 